\title{
Liver/kidney microsomal antibody type 1 targets CYP2D6 on hepatocyte plasma membrane
}

L Muratori, M Parola, A Ripalti, G Robino, P Muratori, G Bellomo, R Carini, M Lenzi, M P Landini, E Albano, F B Bianchi

\begin{abstract}
Background-Liver/kidney microsomal antibody type 1 (LKM1) is the marker of type 2 autoimmune hepatitis (AIH) and is detected in up to $6 \%$ of patients with hepatitis $\mathrm{C}$ virus $(\mathrm{HCV})$ infection. It recognises linear and conformational epitopes of cytochrome P450IID6 (CYP2D6) and may have liver damaging activity, provided that CYP2D6 is accessible to effector mechanisms of autoimmune attack.

Methods-The presence of LKM1 in the plasma membrane was investigated by indirect immunofluorescence and confocal laser microscopy of isolated rat hepatocytes probed with 10 LKM1 positive sera (five from patients with AIH and five from patients with chronic $\mathrm{HCV}$ infection) and a rabbit polyclonal anti-CYP2D6 serum.

Results-Serum from both types of patient stained the plasma membrane of non-permeabilised cells, where the fluorescent signal could be visualised as discrete clumps. Conversely, permeabilised hepatocytes showed diffuse submembranous/cytoplasmic staining. Adsorption with recombinant CYP2D6 substantially reduced plasma membrane staining and LKM1 immunoblot reactivity. Plasma membrane staining of LKM1 colocalised with that of anti-CYP2D6. Immunoprecipitation experiments showed that a single $50 \mathrm{kDa}$ protein recognised by anti-CYP2D6 can be isolated from the plasma membrane of intact hepatocytes.

Conclusions-AIH and HCV related LKM1 recognise CYP2D6 exposed on the plasma membrane of isolated hepatocytes. This observation supports the notion that anti-CYP2D6 autoreactivity may be involved in the pathogenesis of liver damage.
\end{abstract}

Department of Internal Medicine, Cardioangiology, Hepatology, University of Bologna, Bologna,

Italy

L Muratori

P Muratori

M Lenzi

F B Bianchi

Department of Clinical and Experimental

Medicine, Section of

Microbiology

A Ripalti

M P Landini

Department of

Medicine and

Experimental

Oncology, University

of Turin, Turin, Italy

M Parola

G Robino

Department of Medical Sciences, University " $A$ Avogadro" of East Piedmont, Novara, Italy

G Bellomo

R Carini

E Albano

Correspondence to: Dr L Muratori,

Dipartimento di Medicina Interna, Cardioangiologia, Epatologia, Università di Bologna, Policlinico S Orsola, via Massarenti 9, 40138 Bologna, Italy

Accepted for publication 5 October 1999
(Gut 2000;46:553-561)

Keywords: liver/kidney microsomal antibody type 1; autoimmunity; autoimmune hepatitis; hepatitis $\mathrm{C}$ virus infection; confocal microscopy

Liver/kidney microsomal antibody type 1 (LKM1) is the serological hallmark of type 2 autoimmune hepatitis (AIH), ${ }^{1}$ a severe form of autoimmune liver disease which usually affects children, runs a poor course without adequate immunosuppression, and often requires liver transplantation. ${ }^{2}{ }^{3}$ LKM1 is also present in up to $6 \%$ of patients chronically infected with hepatitis $\mathrm{C}$ virus (HCV). ${ }^{4}$
A basic fundamental requirement to hypothesise a role for any autoreactivity in the development and progression of immunologically mediated, organ specific damage is the physical accessibility of the target antigen(s) in the affected organ by the supposed effector mechanisms - that is, autoantibodies and autoreactive $\mathrm{T}$ cells. LKM1 is suggested to have direct liver damaging activity, but evidence on the presence of its target antigen on the hepatocyte plasma membrane-a prerequisite for antibody accessibility-is so far inconclusive. $^{5}$

The main LKM1 autoantigen cytochrome P450IID6 (CYP2D6) ${ }^{6}$ belongs to the P450 cytochrome family. CYP2D6, as well as the other isoforms of cytochrome P450, are largely located in the hepatocyte endoplasmic reticulum. However, with this subcellular distribution, CYP2D6 would not be available as a target of a hypothetical liver specific autoimmune process. A growing body of evidence indicates that a massive flow of vesicles from the endoplasmic reticulum through the Golgi apparatus to the plasma membrane ${ }^{78}$ transports endoluminal or membrane bound proteins, including cytochrome P450s, to the plasma membrane. ${ }^{9}$ Several isoforms of cytochrome P450, ${ }^{10-13}$ including CYP2D6, ${ }^{14}{ }^{15}$ may be exposed on the outer surface of the hepatocyte plasma membrane. However, the presence of CYP2D6 on the hepatocyte plasma membrane is controversial, as studies using LKM1 positive autoimmune sera failed to show its presence on the hepatocyte surface. ${ }^{16}{ }^{17}$ The question of LKM1 involvement in immunologically mediated liver damage therefore remains open. ${ }^{18}$

\section{Methods}

PATIENTS AND SERA

LKM1 positive sera were obtained from five patients suffering from type $2 \mathrm{AIH}$ and from five patients with HCV related chronic hepatitis C (table 1). All five autoimmune patients fulfilled internationally agreed criteria for the diagnosis of "definite" autoimmune hepatitis, ${ }^{19}$ and were all negative for serum HCV RNA sequences by "nested" reverse transcription-polymerase chain reaction. The

Abbreviations used in this paper: LKM1, liver/kidney microsomal antibody type $1 ; \mathrm{AIH}$, autoimmune hepatitis; $\mathrm{HCV}$, hepatitis $\mathrm{C}$ virus; CYP2D6, cytochrome P450IID6; SMA-T, smooth muscle antibodies with a peritubular pattern; LC1, liver cytosol antibody type 1; PBS, phosphate buffered saline; SDS/PAGE, sodium dodecyl sulphate/polyacrylamide gel electrophoresis; TBS, Tris buffered saline. 
Table 1 Clinical, immunological, and virological features of 10 LKM1 positive patients

\begin{tabular}{lllllllll}
\hline Patient & Sex & Age $(y)$ & Diagnosis & IAHG score & $\begin{array}{l}\text { Recombinant } \\
\text { CYP2D6 }\end{array}$ & $\begin{array}{l}\text { Native } \\
\text { CYP2D6 }\end{array}$ & LKM1 titre & HCVRNA \\
\hline 1 & F & 26 & Type 2 AIH & $19(\mathrm{~d})$ & Positive & Positive & $<1: 4000$ & Negative \\
2 & F & 8 & Type 2 AIH & $20(\mathrm{~d})$ & Positive & Positive & $<1: 4000$ & Negative \\
3 & F & 26 & Type 2 AIH & $18(\mathrm{~d})$ & Positive & Positive & $1: 2000$ & Negative \\
4 & F & 18 & Type 2 AIH & $18(\mathrm{~d})$ & Positive & Positive & $1: 1000$ & Negative \\
5 & M & 23 & Type 2 AIH & $17(\mathrm{~d})$ & Positive & Positive & $1: 500$ & Negative \\
6 & M & 18 & HCV related & 7 & Positive & Positive & $<1: 4000$ & Positive \\
7 & F & 32 & HCV related & 7 & Positive & Positive & $<1: 4000$ & Positive \\
8 & F & 60 & HCV related & 5 & Positive & Positive & $1: 2000$ & Positive \\
9 & F & 29 & HCV related & 5 & Positive & Positive & $1: 1000$ & Positive \\
10 & F & 32 & HCV related & 6 & Positive & Positive & $1: 500$ & Positive \\
\hline
\end{tabular}

$\mathrm{AIH}$, autoimmune hepatitis; IAHG, International Autoimmune Hepatitis Group; d, "definite” diagnosis of AIH; LKM1, liver/kidney microsomal antibody type 1 .

typical LKM1 pattern was detectable by indirect immunofluorescence on cryostat rat liver and kidney sections. ${ }^{20}$ In addition, all sera recognised a $50 \mathrm{kDa}$ microsomal protein extracted from human liver microsomes, prokaryotically expressed recombinant human CYP2D 6 by Western immunoblotting, ${ }^{21}$ and eukaryotically expressed CYP2D6 by immunoprecipitation. ${ }^{22}$

As controls, we used sera from three patients with chronic hepatitis $\mathrm{C}$ negative for LKM1 reactivity and sera of three healthy subjects. Further controls were performed with serum from a patient with type $1 \mathrm{AIH}$, positive for smooth muscle antibodies with peritubular pattern (SMA-T), ${ }^{23}$ and serum from a patient with type 2 AIH positive for liver cytosol antibody type 1 (LC1). ${ }^{24}$ Both these sera were also negative for LKM1 and anti-CYP2D6 reactivity.

ISOLATED HEPATOCYTE PREPARATIONS

Male Sprague-Dawley rats (170-220 g) were supplied by Harlan-Nossan (Corezzana, Italy) and were used for all the experiments. Rat hepatocytes were isolated by collagenase liver perfusion as previously described. ${ }^{25}$ Dead hepatocytes and cell debris were removed by spinning the cell suspension ( $1 \mathrm{~min}$ at $1000 \mathrm{~g}$ ) through a $5 \mathrm{ml}$ layer of $37.5 \%$ Percoll (Pharmacia, Uppsala, Sweden) solution ( $\mathrm{d}=$ 1.06) in Hanks buffer, pH 7.4. The hepatocytes were finally resuspended at a concentration of $2.5 \times 10^{5}$ cells $/ \mathrm{ml}$ in a modified Krebs/Henseleit/Hepes buffer, pH 7.4, containing $120 \mathrm{mmol} / 1 \mathrm{NaCl}, 5 \mathrm{mmol} / 1 \mathrm{KCl}, 1$ $\mathrm{mmol} / 1 \mathrm{CaCl}_{2}, 2 \mathrm{mmol} / 1 \mathrm{MgSO}_{4}, 1 \mathrm{mmol} / 1$ $\mathrm{NaHPO}_{4}$ and $25 \mathrm{mmol} / \mathrm{l}$ Hepes.

DETERMINATION OF HEPATOCYTE VIABILITY Cell viability was estimated by Trypan blue exclusion. At the beginning of the experiments, hepatocyte viability was about $90 \%$.

\section{IMMUNOFLUORESCENCE ANALYSIS}

For immunofluorescence analysis, hepatocytes (250 000 cells $/ \mathrm{ml}$ ) were allowed to adhere to collagen type IV coated glass coverslips placed in six-well cell culture plates for a one hour incubation at $37^{\circ} \mathrm{C}$ in a humidified chamber. At the end of the incubation, the coverslips were rinsed briefly with the incubation medium and fixed for 10 minutes in freshly prepared buffered $4 \%(\mathrm{v} / \mathrm{v})$ formaldehyde solution in phosphate buffered saline (PBS) $\mathrm{pH}$ 7.4. Fixed cells were rinsed in PBS containing 3\% (w/v) bovine serum albumin. For some experiments isolated hepatocytes were permeabilised in PBS containing $0.1 \%(\mathrm{v} / \mathrm{v})$ Triton X-100.

After fixation, the coverslips were covered with $1 \mathrm{ml} 10 \%$ ( $/ \mathrm{v}$ ) calf serum solution in PBS and incubated for one hour at room temperature. The reaction with the primary antibodies was performed by incubating the coverslips for 30 minutes at room temperature with LKM1 positive sera diluted 1:50-1:200 in $1 \mathrm{ml}$ PBS containing $1 \%$ bovine serum albumin (PBS/BSA). The coverslips were then washed three times with PBS/BSA and further incubated with the secondary antibody consisting of fluorescein labelled goat anti-human IgG (Sigma Chemical Co, St Louis, Missouri, USA; dilution 1:200) in PBS/BSA. Unbound antibodies were removed by washing the coverslips three times with $2 \mathrm{ml}$ PBS. The coverslips were finally mounted on to glass slides using Vectashield mounting medium (Vector Laboratories Inc, Burlingame, California, USA).

For double labelling experiments, the cells were incubated with polyclonal anti-CYP2D6 rabbit serum raised against the whole CYP2D6 protein (Gentest, Woburn, Massachusetts, USA; diluted 1:100) and with LKM1 positive sera, as described above. The cells were then stained with secondary antibodies consisting of goat anti-rabbit IgG conjugated with Texas Red (Molecular Probes, Eugene, Oregon, USA; diluted 1:250) and fluorescein labelled goat anti-human IgG (diluted 1:150). Immunological cross reactivity between goat anti-rabbit IgG and human immunoglobulin and between goat anti-human IgG and rabbit immunoglobulin was negligible.

The fluorescence localisation was determined with a laser scanner confocal microscope Bio-Rad MRC 600 (Bio-Rad Instruments, Watford, Middlesex, UK) equipped with a Nikon Diaphot TMD-2S inverted fluorescence microscope (Nikon, Tokyo, Japan) and a Nikon Plan 60/1.40 oil immersion lens. Nine to twelve focal frames, depending on the cell thickness, were taken along the $\mathrm{z}$ axis at $1 \mu \mathrm{m}$ intervals and then merged to obtain reconstructed images.

To improve the characterisation of the intracellular localisation, single median focal frames of $1 \mu \mathrm{m}$ thickness were also collected. All images were obtained using the same conditions of setting. In particular, black level was set at 5.4 for red fluorescence and at 5.55 for green 
fluorescence; gain level was 9.8 for both. No electronic modification of background cut off was used.

EXPRESSION AND PURIFICATION OF HUMAN RECOMBINANT CYP2D6

$\lambda$ gt11 genomic DNA was extracted from a phage clone containing a $1.2 \mathrm{~kb}$ insert coding for CYP2D6 (a gift from Dr Yun Ma, UCL, London, UK). Phage propagation was by standard methods ${ }^{26}$ and DNA was purified with a commercial kit (Quick!Spin; Bio101 Inc, Vista, California, USA). The CYP2D6 insert was amplified using 5 ng purified phage DNA as a template and a primer pair with homology to regions immediately upstream and downstream of the phage cloning site (forward 5'-TGGCGACGACTCCTGGAGCC CG-3'; reverse 5'-TGACACCAGACCAACT GGTAATGG-3'). A Rapid cycler (Idaho Technology, Idaho Falls, Idaho, USA) was used for the reaction, and the amplification was carried out through 30 cycles as follows: denaturation at $98^{\circ} \mathrm{C}$ for 0 seconds, annealing at $56^{\circ} \mathrm{C}$ for 0 seconds and extension at $72^{\circ} \mathrm{C}$ for 80 seconds. The polymerase chain reaction product containing the CYP2D6 insert was then purified, digested with EcoRI, filled in, and subcloned in pROS (a prokaryotic expression vector downstream of lacZ) following standard procedures. ${ }^{26}$ Ligated plasmid DNA from the pROS/CYP2D6 construct was transfected in Escherichia coli cells (DH5a), and transformed cells were isolated on selective agar plates containing ampicillin. Some colonies were isolated and analysed for expression of the fusion protein; after exponential growth in liquid culture medium, expression was induced by adding isopropyl $\beta$-Dthiogalactoside $(5 \mathrm{mmol} / \mathrm{l})$. After four hours, cells were collected and lysates were prepared and analysed by sodium dodecyl sulphate/ polyacrylamide gel electrophoresis (SDS/ PAGE; $7.5 \%$ gel). Coomassie staining of the gel allowed visualisation of the induced protein as a $91 \mathrm{kDa}$ polypeptide. After transblotting on a nitrocellulose sheet, immunoblot experiments were performed with a monoclonal antibody specific for human CYP2D6 (BII $/ 7^{27}$; a gift from Professor Urs A Meyer, Basel University, Basel, Switzerland) which strongly reacted with the $91 \mathrm{kDa}$ fusion protein. This fusion protein encodes amino acids $124-497$ of CYP2D6, corresponding to the most immunoreactive portion of the protein. To purify recombinant CYP2D6, preparative SDS/ PAGE was performed with the bulk of the isopropyl $\beta$-D-thiogalactoside induced pROS/ CYP2D6 transformed $E$ coli lysates; protein bands were visualised by negatively staining gels with $0.3 \mathrm{M} \mathrm{CuCl}_{2}$, the $91 \mathrm{kDa}$ band was excised, and the protein was electroeluted in SDS running buffer. The eluted protein was finally extensively dialysed against PBS.

ADSORPTION OF THE LKM1 POSITIVE SERA WITH RECOMBINANT CYP2D6

LKM1 positive sera (dilution of $1: 40$ ) were incubated overnight on a rotor wheel in PBS containing $0.1 \mathrm{mg} / \mathrm{ml}$ purified recombinant
CYP2D6. After centrifugation for 10 minutes at $14000 \mathrm{rpm}$ in a bench centrifuge, the pellet was discarded and the supernatant was tested in immunofluorescence experiments on cryostat sections of rat liver and kidney sections. Adsorbed sera were diluted to a final concentration of 1:200 and tested by immunoblotting on human liver microsomes and recombinant human CYP2D6. Finally, adsorbed sera were also tested at a dilution of $1: 100-1: 200$ on isolated rat hepatocytes and analysed by confocal laser microscopy.

IMMUNOBLOT EXPERIMENTS WITH HUMAN SERA Human liver microsomal proteins were prepared as previously described. ${ }^{28}$ Microsomal protein or purified recombinant CYP2D6 (20 $\mu \mathrm{g}$ or $10 \mathrm{ng}$ respectively per lane) were separated by SDS/PAGE in $7.5 \%$ polyacrylamide minigels (Mini-Protean II System; BioRad Laboratories, Richmond, California, USA) and transblotted on to nitrocellulose filters, which were then incubated in blocking solution (Tris buffered saline (TBS) containing $3 \%$ bovine serum albumin) for one hour at room temperature. The filters were then cut into strips, and each strip was incubated overnight at $4^{\circ} \mathrm{C}$ with non-adsorbed and preadsorbed LKM1 positive sera (final dilution 1:200). After incubation, the strips were washed three times in TBS containing $0.1 \%$ Tween 20, and subsequently incubated for two hours at room temperature in blocking solution containing peroxidase conjugated rabbit antihuman IgG (dilution 1:1000; Dako, Copenhagen, Denmark) as secondary antibody. After further washing, the colorimetric reaction was developed with 4-chloro-1-naphthol for 10 minutes at room temperature.

IMMUNOPRECIPITATION OF ANTIGENS RECOGNISED BY HUMAN LKM1 POSITIVE SERA ON HEPATOCYTE PLASMA MEMBRANES

IgG from autoimmune and $\mathrm{HCV}$ related LKM1 positive human sera were allowed to bind to Protein A-Sepharose (Sigma Chemical

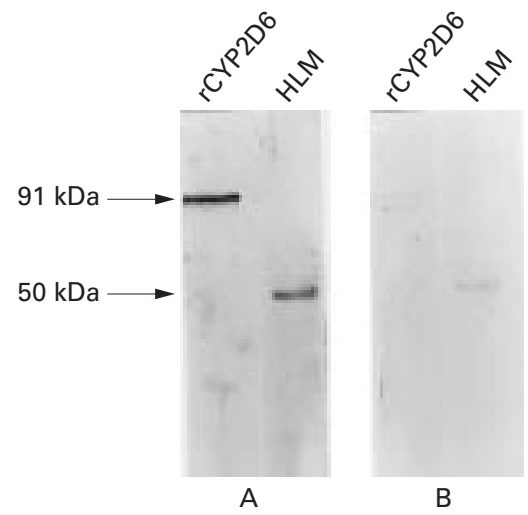

Figure 1 Immunoblotting reactivity of a representative liver/kidney microsomal antibody type 1 (LKM1) positive serum with recombinant human CYP2D6 ( $r C Y P 2 D 6)$ and human liver microsomes (HLM). Serum reacts with a $91 \mathrm{kDa}$ polypeptide, corresponding to $\mathrm{rCYP2D6}$ expressed in Escherichia coli and with a $50 \mathrm{kDa}$ polypeptide corresponding to native human CYP2D6 (A). After preadsorption with $r C Y P 2 D 6$, the reactivity with rCYP2D6 is completely lost, whereas a faint $50 \mathrm{kDa}$ band is still visible with native CYP2D6 (B). 
Co) by incubating the sera overnight at $4^{\circ} \mathrm{C}$ with $20 \mathrm{mmol} / \mathrm{l}$ Tris/ $\mathrm{HCl}$ buffer, $\mathrm{pH}$ 7.4, containing $150 \mathrm{mM} \mathrm{NaCl}, 5 \mathrm{mM}$ EDTA, $1 \%$ Triton X-100, $1 \mathrm{mM}$ phenylmethanesulphonyl fluoride, and $0.05 \%$ aprotinin (RIPA-PTK buffer). Sepharose beads were then centrifuged at $14000 \mathrm{rpm}$ for one minute, and two additional washings with PBS were performed to eliminate unbound antibodies and RIPAPTK buffer. The beads resuspended in $200 \mu \mathrm{l}$ Krebs/Henseleit/Hepes buffer, pH 7.4, were added to isolated hepatocyte suspensions $(0.25$ $\times 10^{6}$ cells $/ \mathrm{ml}$ ) and incubated for 20 minutes at $20^{\circ} \mathrm{C}$ with gentle shaking on a rocking platform.

The cells were centrifuged at $400 \mathrm{~g}$ for three minutes, and two additional washings with PBS at $400 \mathrm{~g}$ for three minutes were performed to remove any unbound beads. The hepatocytes were lysed by 45 minutes of incubation in ice cold RIPA-PTK buffer. The beads attached
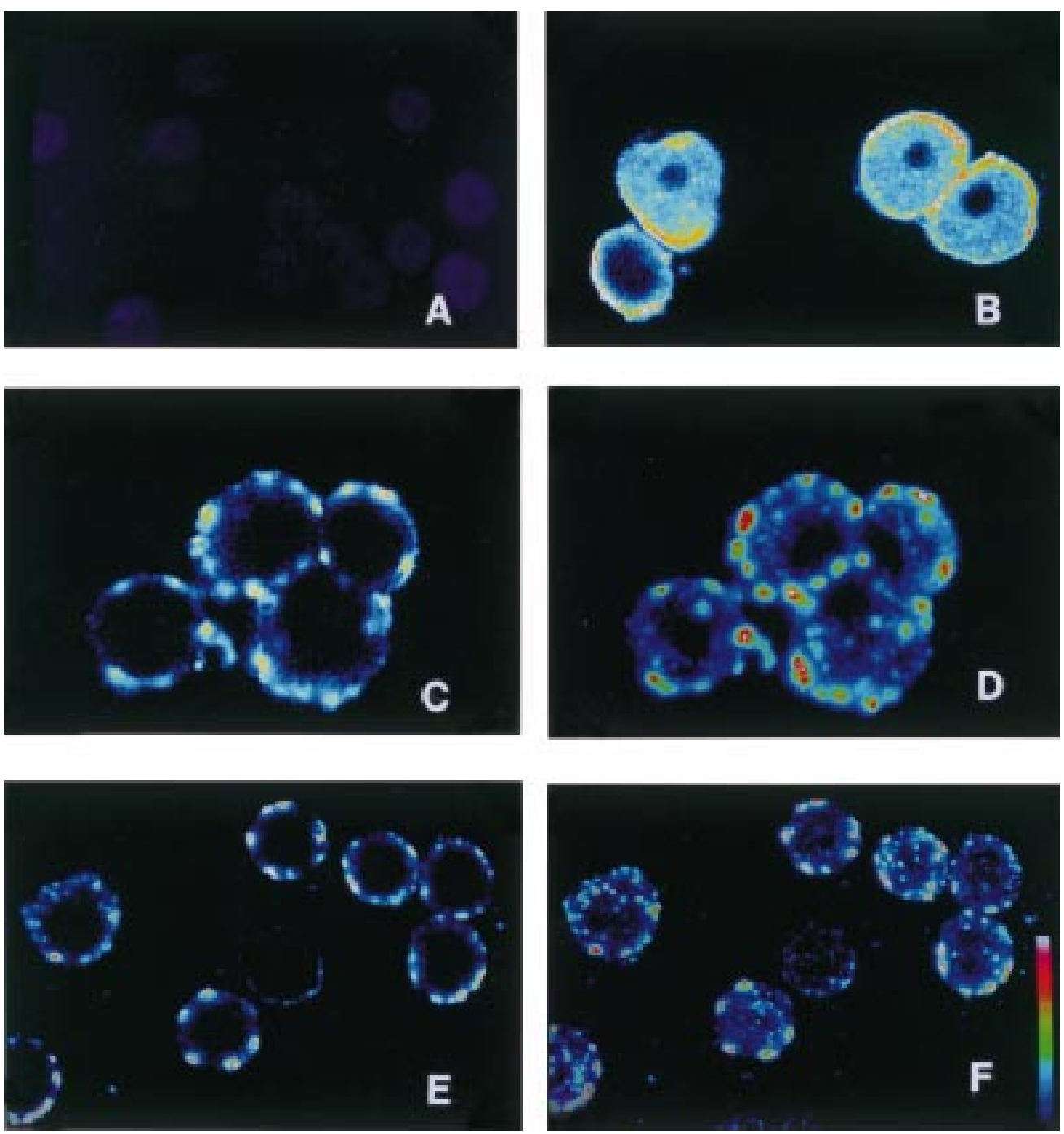

Figure 2 Confocal microscope images of plasma membrane staining by liver/kidney microsomal antibody type 1 (LKM1) positive sera in freshly isolated rat hepatocytes. The hepatocytes were probed with LKM1 positive sera (diluted 1:50) and fluorescein labelled goat anti-human IgG (diluted 1:200). The images correspond to: $(A)$ non-permeabilised hepatocytes exposed to the serum of a healthy control; (B) permeabilised hepatocytes probed with the serum of a LKM1 positive subject; $(C-F)$ non-permeabilised hepatocytes probed with LKM1 positive sera from a patient with AIH (C,D) or a patient with chronic HCV infection $(E, F)$. Images $D$ and $F$ were obtained by merging nine focal frames taken along the $z$ axis at $1 \mu m$ intervals. Images $B, C$, and $E$ show the focal frame corresponding to the median optical section of the hepatocytes.

Fluorescence is shown in pseudocolours increasing from blue to red and white. Original magnifications are $30 \times$ for images $A, B, E$, and $F$ and $60 \times$ for images $C$ and $D$.

to plasma membrane fragments were precipitated by centrifugation at $14000 \mathrm{rpm}$ for one solubilised in $0.1 \mathrm{ml}$ Laemmli's buffer and boiled for three minutes to separate the Sepharose beads. Aliquots of $1 \mu \mathrm{g}$ protein were blotted on nitrocellulose filters (Amers then Life Science, Amersham, Buckinghamshire, UK), by using a Bio-Rad transblot device and a transfer buffer containing $20 \%$ methanol, 48 filters were then incubated at $4^{\circ} \mathrm{C}$ overnight in TBS containing $5 \%$ low fat milk $/ 0.05 \%$ Tween 20 , to block non-specific binding, and subsequently exposed for one hour at $37^{\circ} \mathrm{C}$ to either (1:100 dilution) or goat anti-glucose-6phosphatase antibodies (kindly provided by Dr A Burchell, Dundee, Scotland, UK; 1:1000

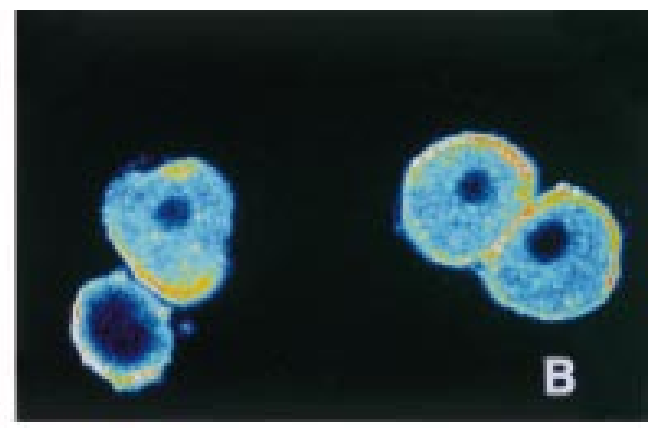




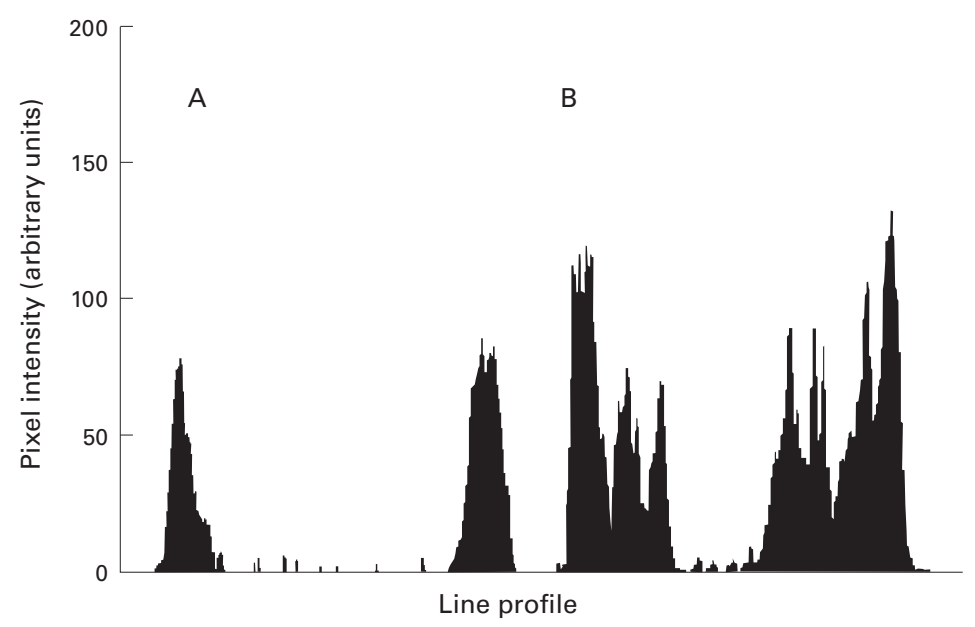

Figure 3 Frequency distribution of the pixels referring to cellular fluorescence in optical sections from hepatocytes stained with liver/kidney microsomal antibody type 1 (LKM1) positive serum (diluted 1:40) and fluorescein labelled goat anti-human IgG (diluted 1:200). (A) Typical distribution in non-permeabilised hepatocytes; (B) distribution in hepatocytes that were permeabilised before fixation as reported in the Methods section.

dilution) in TBS containing $2 \%$ milk and $0.05 \%$ Tween 20. Filters were washed four times with TBS containing $0.05 \%$ Tween 20 and then incubated with the anti-rabbit horseradish peroxidase conjugated antibodies or anti-goat horseradish peroxidase conjugated antibodies (1:5000 or 1:10.000 dilution respectively) in TBS containing $2 \%$ milk and $0.05 \%$ Tween 20 . After the incubation, filters were again washed four times with TBS containing $0.05 \%$ Tween 20 , and immunoreactive proteins were detected by the enhanced chemiluminescence plus kit (Amersham Life Science) on an $x$ ray film (Eastman-Kodak, Rochester, New York, USA).

In some experiments bound antibodies were stripped from nitrocellulose filters which were then reprobed with a different antibody. For this, membranes were incubated at $50^{\circ} \mathrm{C}$ for 30-40 minutes with occasional agitation in stripping buffer $(100 \mathrm{mmol} / \mathrm{l}$ 2-mercaptoethanol; $2 \%$ SDS; $62.5 \mathrm{mmol} / 1 \mathrm{Tris} / \mathrm{HCl} \mathrm{pH}$ 6.7), then washed three times for 15 minutes using large volumes of TBS containing $0.05 \%$ Tween 20. This procedure allowed the complete removal of both primary and secondary antibodies. The observed loss of protein in the filters after stripping was usually less then $10 \%$. The usual blocking and probing steps followed.

\section{Results}

Reactivity of LKM1 positive sera with surface antigen(s) of freshly isolated rat hepatocytes was investigated by indirect immunofluorescence and confocal laser microscopy using the sera from five patients with type $2 \mathrm{AIH}$ and five patients with LKM1 positive chronic HCV infection. All the sera showed the typical LKM1 pattern by indirect immunofluorescence on rat liver and kidney tissue sections. When tested in immunoblots, the same sera recognised a $50 \mathrm{kDa}$ polypeptide using human liver microsomes as substrate, and specifically reacted with recombinant CYP2D6 (table 1 and fig 1).

Immunostaining of non-permeabilised hepatocytes with LKM1 positive sera and fluorescein conjugated goat anti-human IgG resulted in extensive fluorescence labelling of the cells. At high magnification, discrete distribution of the fluorochrome on the hepatocyte surface was observed in the absence of any cytoplasmic staining (fig 2). No appreciable difference in the fluorescence pattern was observed with LKM1 positive sera from patients with type $2 \mathrm{AIH}$ or with LKM1 positive chronic hepatitis $\mathrm{C}$, while no fluorescence labelling was obtained using sera from healthy controls. The plasma membrane localisation of the epitopes recognised by LKM1 antibodies was confirmed by the analysis of sequential focal frames taken at $1 \mu \mathrm{m}$ intervals which showed a ring distribution of the fluorescence on the cell surface. This pattern was very different from the diffuse submembranous/ cytoplasmic staining observed when the same sera were allowed to react with permeabilised hepatocytes. Furthermore, the analysis of pixel intensity distributions in a series of focal sections taken at the same level in nonpermeabilised hepatocytes stained with LKM1 positive sera showed two narrow peaks corresponding to the cell surface (fig 3A). Conversely, a much broader pixel distribution leaving an empty area corresponding to the cell nucleus was appreciable in permeabilised
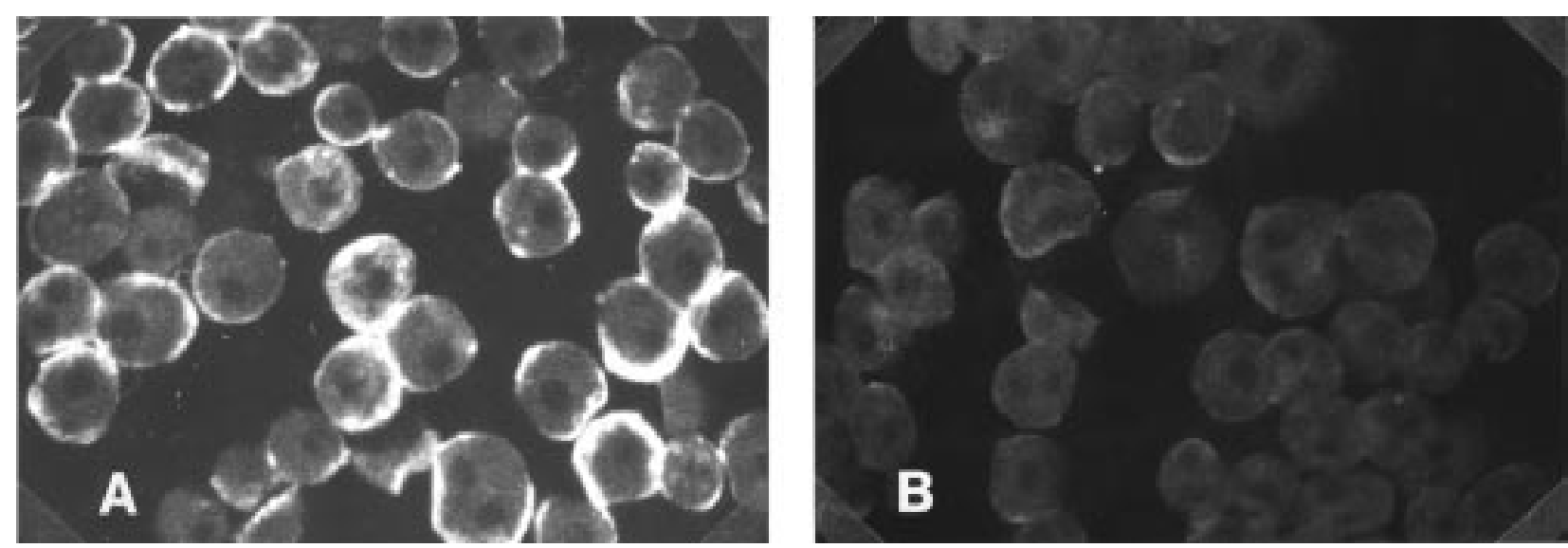

Figure 4 Effect of the preadsorption of liver/kidney microsomal antibody type 1 (LKM1) positive sera with recombinant human CYP2D6 on the surface staining of isolated rat hepatocytes. LKM1 sera were preadsorbed as described in the Methods section with human recombinant CYP2D6.

Non-permeabilised rat hepatocytes were stained with native $(A)$ and preadsorbed $(B)$ sera at the same dilution (1:200). Original magnification $\times 10$. 

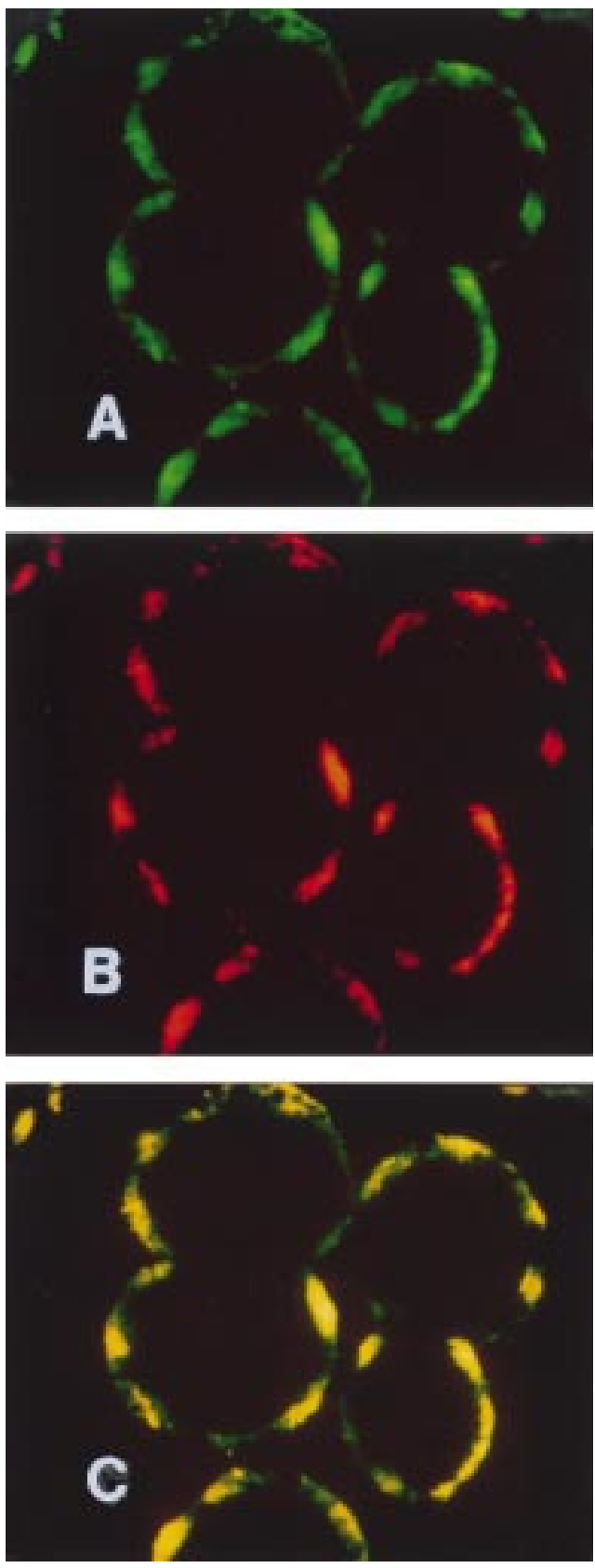

Figure 5 Confocal microscope images of the same focal section of adjacent rat hepatocytes allowed to react with rabbit anti-CYP2D6 serum and liver/kidney microsomal antibody type 1 (LKM1) positive human serum. The cell preparation was double stained with goat anti-human IgG labelled with fluorescein and with goat anti-rabbit IgG complexed with Texas Red. The same median cell optical section shows the green fluorescence due to fluorescein in $(A)$ and the red fluorescence of Texas Red in (B). (C) Result of the electronic merging of images $(A)$ and $(B)$. Original magnification $\times 30$.

hepatocytes (fig 3B). A similar diffuse cytoplasmic fluorescence, but without any labelling of the plasma membranes, was observed when the sera from two patients with $\mathrm{AIH}$, one of which had high titres of SMA-T and the other had high titres of LC1, were used for immunofluorescence staining of both permeabilised and non-permeabilised cells.

Adsorption of the sera from patients infected with $\mathrm{AIH}$ or $\mathrm{HCV}$ with $0.1 \mathrm{mg} / \mathrm{ml}$ human recombinant CYP2D6 removed the immuno- blotting reaction with recombinant CYP2D6 and substantially reduced the reactivity with the $50 \mathrm{kDa}$ band when human liver microsomal proteins were used as antigenic substrate (fig 1). CYP2D6 adsorbed sera did not display any appreciable immunostaining of hepatocyte plasma membranes when allowed to react with non-permeabilised hepatocytes (fig 4).

Staining of non-permeabilised hepatocytes with a rabbit anti-CYP2D6 polyclonal serum gave a cell surface fluorescence distribution similar to that of LKM1 positive sera, and double labelling experiments with LKM1 positive sera (green fluorescence) and rabbit anti-CYP2D6 (red fluorescence) showed colocalisation of their targets. Confocal microscope examination of the same cells for both green and red surface fluorescence disclosed an indistinguishable pattern (fig 5A,B), and electronic merging of the two images showed that cell surface spots reacting with LKM1 positive sera colocalised with those recognised by rabbit anti-CYP2D6 (fig 5C).

To confirm further CYP2D6 plasma membrane localisation, antigens located on the outer surface of plasma membranes were immunoprecipitated by exposing intact hepatocytes to LKM1 IgG coated Protein A-Sepharose beads. After removal of unbound Sepharose, the cells were lysed, and plasma membrane fragments bound to Sepharose particles were precipitated by centrifugation. The resulting precipitates were then subjected to SDS/PAGE under non-reducing conditions and the Western blot filters stained using either rabbit anti-CYP2D6 or a polyclonal serum against glucose-6-phosphatase. Figure 6 shows that anti-CYP2D6 serum stained a single band at $50 \mathrm{kDa}$ when plasma membranes were immunoprecipitated with human LKM1 positive serum that was either autoimmune (lanes 2 and 3) or HCV related (lanes 4 and 5). No staining was observed when Protein A-Sepharose alone was used for immunoprecipitation (lane 1). When similar filters were probed with anti-glucose-6-phosphatase antibodies, a very faint band of about $38 \mathrm{kDa}$ was observed, indicating negligible contamination of microsomal proteins.

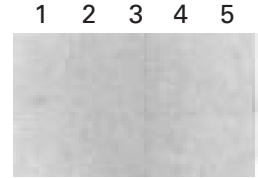

G6Pase

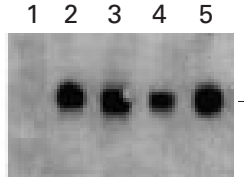

CYP2D6
Figure 6 Immunoprecipitation of antigens recognised by human liver/kidney microsomal antibody type 1 (LKM1) sera on hepatocyte plasma membranes. Plasma membrane antigens of isolated hepatocytes were immunoprecipitated by using human LKM1 positive sera from either autoimmune or HCV related hepatitis patients complexed with Protein A coated Sepharose beads. The precipitated proteins were submitted to SDS/PAGE and visualised using a rabbit polyclonal anti-human CYP2D6 serum. Lane 1, control beads without attached LKM1 serum; lanes 2 and 3, two different LKM1 positive sera from patients with autoimmune hepatitis; lanes 4 and 5, two different LKM1 positive sera from patients with hepatitis $C$ virus (HCV) hepatitis. G6Pase, glucose-6-phosphatase. 


\section{Discussion}

In this study, we show that LKM1 positive sera react with discrete clumps on the outer plasma membrane surface of non-permeabilised freshly isolated rat hepatocytes and provide several lines of evidence indicating that the antigenic target of LKM1 reactivity on the liver cell membrane is CYP2D6. Firstly, cell surface staining is observed only with LKM1 positive sera, and not with control sera containing unrelated autoantibodies such as SMA-T and LC1; secondly, the plasma membrane staining is specifically abolished by adsorption with recombinant human CYP2D6; thirdly, double staining experiments show that anti-CYP2D6 raised in rabbit colocalises on the plasma membrane with LKM1 signal; fourthly, LKM1 coated beads specifically immunoprecipitate isolated hepatocytes through binding of a 50 $\mathrm{kDa}$ plasma membrane protein which is recognised by rabbit anti-CYP2D6.

The expression of surface antigens recognised by LKM1 positive sera was first observed by Lenzi and co-workers ${ }^{29}$ using indirect immunofluorescence staining of freshly isolated rat hepatocytes, well before the identification and characterisation of CYP2D6 as the LKM1 molecular target. This finding was confirmed by two additional papers showing LKM1 positive sera reacting with the plasma membranes of either isolated $\mathrm{rat}^{14}$ or human ${ }^{15}$ hepatocytes. Two other papers, however, reached opposite conclusions. ${ }^{16}{ }^{17}$ In particular, Yamamoto and co-workers ${ }^{17}$ were unable to detect plasma membrane immunofluorescence staining of cultured rat and human hepatocytes by the whole serum of two AIH patients as well as by affinity purified antibodies against the 254-271 amino acid peptide of CYP2D6, the main linear epitope recognised by autoimmune LKM1 positive sera. No explanation has been produced for these conflicting reports, and since then the issue as to the presence of the LKM1 target on the surface of hepatocyte plasma membrane has remained unsettled.

At variance with previous studies using whole and uncharacterised LKM1 positive sera $^{1429}$ or affinity purified LKM1 antibodies, ${ }^{15}{ }^{17}$ we used extensively characterised LKM1 positive sera and a polyclonal antiCYP2D6 antibody in double staining and immunoprecipitation experiments.

The discrepancies between our findings and those of Yamamoto and co-workers ${ }^{17}$ may be due to our use of freshly isolated rat hepatocytes instead of 24 hour cultured cells. Snap frozen rat liver sections are the conventional antigenic substratum for LKM1 detection, because rat hepatocytes express CYP2D1, the rat orthologue of human CYP2D6. In view of the fact that cytochrome P450 levels decrease by $60-70 \%$ during short term culture of freshly isolated hepatocytes, ${ }^{30}{ }^{31}$ and that the plasma membrane content of cytochrome P450 is about $10 \%$ of that in microsomes, ${ }^{15}$ it is possible that CYP2D6 plasma membrane levels have fallen below immunofluorescence detection limits after a 24 hour cell culture.

The observation that normal hepatocytes express CYP2D6 on the outer surface of the plasma membrane is not completely unexpected, as several P450 isoforms have already been described on the hepatocyte surface $^{8111415}$ and in highly purified secretory fractions of Golgi apparatus obtained from rat hepatocytes. ${ }^{9} \quad$ Moreover, Loeper and co-workers $^{32}$ have recently reported that human CYP2D6 expressed in yeast is exposed on the external aspect of the plasma membrane where it is functionally competent.

Cloning and expression in bacteria of recombinant CYP2D6 allowed the study of LKM1 reactivity in both AIH positive and HCV positive sera. While nearly all AIH LKM1 positive sera recognise CYP2D6, only a fraction of $\mathrm{HCV}$ related LKM1 positive sera do so. ${ }^{21334}$ The interpretation of these findings was that AIH sera more often contain antibodies that react with linear epitopes than do LKM1 positive HCV sera. The latter were thought to recognise mainly the entire protein folded in its native conformation. The recent availability of CYP2D6 expressed in a eukaryotic system allowed the direct demonstration that $\mathrm{AIH}$ related and $\mathrm{HCV}$ related LKM1 positive sera react similarly with conformational CYP2D6 epitopes. ${ }^{22}{ }^{35}$ To cover the whole spectrum of LKM1 reactivity, the sera used in this study, from either AIH or HCV patients, were chosen on the basis of their ability to recognise CYP2D6 extracted from human liver and its prokaryotic and eukaryotic recombinant forms.

The residual weak reactivity of CYP2D6 adsorbed sera in immunoblots against native microsomal preparations is probably due to antibodies recognising linear epitopes in the denatured protein that had not been removed by recombinant CYP2D6. Nonetheless, CYP2D6 adsorbed sera failed to display any significant immunostaining of hepatocyte plasma membranes when incubated with nonpermeabilised hepatocytes. This suggests that the epitopes recognised by LKM1 positive sera on the hepatocyte surface are mostly conformational. It is should be noted that rat CYP2D1 and human CYP2D6 share 82\% amino acid sequence, analogous enzymatic activities, and conserved tertiary structure, ${ }^{36}$ thus the conformational epitopes recognised by LKM1 antibodies on CYP2D1 are probably identical with those present on CYP2D6. Moreover, the main linear epitope recognised by autoimmune LKM1 and spanning CYP2D6 amino acids $257-269$ is conserved in rat CYP2D1 (10 identical and one similar amino acid residues out of 13).

Other cytochrome P450 isoenzymes have been found to be targets for autoimmune response in hepatitis associated with dihydralazine (CYP1A2), tienilic acid (CYP2C9), or halothane treatment (CYP2E1) ${ }^{37-39}$ as well as in alcoholic liver disease (CYP2E1, CYP3A4), ${ }^{40}$ hypersensitivity reactions to aromatic anticonvulsants (CYP3A), ${ }^{41}{ }^{42}$ autoimmune polyendocrine syndrome type I (CYP11A1), ${ }^{43}$ and Addison's disease (CYP17, CYP21). ${ }^{45}$ In addition, plasma membrane cytochrome P450s have been shown to be the target of immuno- 
toxic reactions associated with halothane or tienilic acid induced hepatitis. ${ }^{11-13}$

In organ specific autoimmune diseases such as autoimmune haemolytic anaemia and myasthenia gravis, the presence of autoantigens on the cell membrane enables an autoantibody mediated immune attack to occur. It is thus conceivable that LKM1 is also involved in the causation of liver specific injury. The fact that the target antigen of LKM1 reactivity is expressed on the cell surface of normal hepatocytes suggests that, under physiological circumstances, unresponsiveness or immunological tolerance to CYP2D6 is a necessary and common condition, probably maintained by mechanisms that actively prevent the maturation or the stimulation of autoreactive lymphocytes. The breakdown of the immunological tolerance to CYP2D6 is the hallmark of two different liver diseases, namely type $2 \mathrm{AIH}$, a cryptogenic liver disorder, and LKM1 positive chronic hepatitis $\mathrm{C}$, which is characterised by the presence of circulating HCV. In both conditions, loss of $\mathrm{B}$ and $\mathrm{T}$ cell tolerance to CYP2D6 similarly occurs, as documented by the appearance of LKM1 antibodies ${ }^{46} 47$ and LKM1 specific autoreactive $\mathrm{T}$ cells. ${ }^{48}{ }^{49}$ The demonstration that LKM1, in both $\mathrm{AIH}$ and $\mathrm{HCV}$ infection, reacts with its target antigen exposed on the cell surface of nonpermeabilised normal hepatocytes suggests that similar mechanisms of injury may prevail in vivo leading to perpetuation of liver damage in both conditions.

1 Homberg JC, Abuaf N, Bernard O, et al. Chronic active hepatitis associated with antiliver/kidney microsome antibody type 1: a second type of "autoimmune" hepatitis Hepatology 1987;7:1333-9.

2 Mieli-Vergani G, Vergani D. Progress in pediatric autoimmune hepatitis. Semin Liver Dis 1994;14:282-8.

3 Gregorio GV, Portmann B, Reid F, et al. Autoimmune hepatitis in childhood: a 20-year experience. Hepatology 1997;25:541-7.

4 Cassani F, Cataleta $M$, Valentini $P$, et al. Serum autoantibodies in chronic hepatitis C: comparison with autoimmune hepatitis and impact on the disease profile Hepatology 1997;26:561-6.

5 Choudhuri K, Mieli-Vergani G, Vergani D. Cytochrome P4502D6: understanding an autoantigen. Clin Exp Immunol 1997;108:381-3.

6 Manns MP, Johnson EF, Griffin KJ, et al. Major antigen of liver kidney microsomal autoantibodies in idiopathic autoimmune hepatitis is cytochrome P450db1. F Clin Invest 1989;83:1066-72.

7 Myrset AH, Halvorsen B, Ording E, et al. The time courses of intracellular transport of some secretory proteins of rat liver are not affected by an induced acute phase response. Eur f Cell Biol 1993;60:108-14.

8 Robin MA, Maratrat M, Loeper J, et al. Cytochrome $\mathrm{P} 4502 \mathrm{~B}$ follows a vesicular route to the plasma membrane in cultured rat hepatocytes. Gastroenterology 1995;108 $1110-23$

9 Neve EP, Eliasson E, Pronzato MA, et al. Enzyme-specific transport of rat liver cytochrome P450 to the Golgi apparatus. Arch Biochem Biophys 1996;333:459-65.

10 Wu D, Cederbaum AI. Presence of functionally active cytochrome P-450IIE 1 in the plasma membrane of rat hepatochrome P-450IIE1 in the plasma ner
cytes. Hepatology 1992;15:515-24.

11 Robin MA, Maratrat M, Le Roy M, et al. Antigenic targets in tienilic acid hepatitis. Both cytochrome P450 2C11 and 2C11-tienilic acid adducts are transported to the plasma membrane of rat hepatocytes and recognized by human sera. F Clin Invest 1996;98:1471-80.

12 Robin MA, Le Roy M, Descatoire V, et al. Plasma membrane cytochromes P450 as neoantigens and autoimmune targets in drug-induced hepatitis. F Hepatol 1997;26 23-30

13 Eliasson E, Kenna JG. Cytochrome P450 2E1 is a cell surface autoantigen in halothane hepatitis. Mol Pharmacol 1996;50:573-82.

14 Loeper J, Descatoire V, Maurice M, et al. Presence of functional cytochrome $\mathrm{P}-450$ on isolated rat hepatocyte plasma membrane. Hepatology 1990;11:850-8.
15 Loeper J, Descatoire V, Maurice M, et al. Cytochromes P-450 in human hepatocyte plasma membrane: recognition by several autoantibodies. Gastroenterology 1993;104: 203-16.

16 Trautwein C, Gerken G, Lohr H, et al. Lack of surface expression for the B-cell autoepitope of cytochrome P450 IID6 evidenced by flow cytometry. Z Gastroenterol 1993;31: 225-30

17 Yamamoto AM, Mura C, De Lemos-Chiarandini C, et al. Cytochrome P450IID6 recognized by LKM1 antibody is not exposed on the surface of hepatocytes. Clin Exp Immunol 1993;92:381-90.

18 Vergani D, Mieli-Vergani G. Type II autoimmune hepatitis: the conundrum of cytochrome P450IID6. Clin Exp Immunol 1993;92:367-8.

19 Johnson PJ, McFarlane IG. Meeting report: international autoimmune hepatitis study group. Hepatology 1993;18: 998-1005.

20 Rizzetto M, Swana G, Doniach D. Microsomal antibodies in active chronic hepatitis and other disorders. Clin Exp Immunol 1973;15:331-4.

21 Muratori L, Lenzi M, Ma Y, et al. Heterogeneity of liver/kidney microsomal antibody type 1 in autoimmune hepatitis and hepatitis C virus related liver disease. Gut 1995;37:406-12.

22 Ma Y, Gregorio G, Gaken J, et al. Establishment of a novel radioligand assay using eukaryotically expressed cytochrome P4502D6 for the measurement of liver kidney microsomal type 1 antibody in patients with autoimmune hepatitis and hepatitis C virus infection. F Hepatol 1997;26: 1396-402.

23 Bottazzo GF, Florin-Christensen A, Fairfax A, et al. Classification of smooth muscle autoantibodies detected by immunofluorescence. F Clin Pathol 1976;29:403-10.

24 Martini E, Abuaf N, Cavalli F, et al. Antibody to liver cytosol (anti-LC1) in patients with autoimmune chronic active (anti-LC1) in patients with autoimmune

25 Clot P, Parola M, Bellomo G, et al. Plasma membrane hydroxyethyl radical adducts cause antibody-dependent cytotoxicity in rat hepatocytes exposed to alcohol. Gastroenterology 1997;113:265-76.

26 Sambrook J, Fritsh EF, Maniatis T. Molecular cloning. A laboratory manual. 2nd edn. Cold Spring Harbor: Cold Spring Harbor Laboratory Press, 1989.

27 Zanger UM, Hauri HP, Loeper J, et al. Antibodies against human cytochrome P-450db1 in autoimmune hepatitis type II. Proc Natl Acad Sci USA 1988;85:8256-60.

28 Lenzi M, Fusconi M, Selleri L, et al. Counterimmunoelectrophoresis (CIE) for the detection of anti-liver-kidney microsome (LKM) antibodies in the sera of patients with chronic liver disease. F Immunol Methods 1988;111:253-9.

29 Lenzi M, Bianchi FB, Cassani F, et al. Liver cell surface expression of the antigen reacting with liver-kidney microexpression of the antigen reacting with liver-kidney micro-
somal antibody (LKM). Clin Exp Immunol 1984;55:36-40.

30 Sirica AE, Pitot HC. Drug metabolism and effects of carcinogens in cultured hepatic cells. Pharmacol Rev 1979; 31:205-28

31 Maslansky CJ, Williams GM. Primary cultures and the levels of cytochrome P450 in hepatocytes from mouse, rat, hamster, and rabbit liver. In Vitro 1982;18:683-93.

32 Loeper J, Louerat-Oriou B, Duport C, et al. Yeast expressed cytochrome P450 2D6 (CYP2D6) exposed on the external face of plasma membrane is functionally competent. Mol Pharmacol 1998;54:8-13.

$33 \mathrm{Ma} \mathrm{Y}$, Peakman M, Lobo-Yeo A, et al. Differences in immune recognition of cytochrome P4502D6 by liver kidney microsomal (LKM) antibody in autoimmune hepatitis and chronic hepatitis C virus infection. Clin Exp Immunol 1994;97:94-9.

34 Yamamoto AM, Cresteil D, Homberg JC, et al. Characterization of anti-liver-kidney microsome antibody (antiLKM1) from hepatitis $C$ virus-positive and -negative sera. Gastroenterology 1993;104:1762-7.

35 Yamamoto AM, Johanet C, Duclos-Vallee JC, et al. A new approach to cytochrome CYP2D6 antibody detection in autoimmune hepatitis type-2 (AIH-2) and chronic hepatitis $\mathrm{C}$ virus (HCV) infection: a sensitive and quantitative radioligand assay. Clin Exp Immunol 1997;108:396-400.

36 Lewis DF, Eddershaw PJ, Goldfarb PS, et al. Molecular modelling of cytochrome P4502D6 (CYP2D6) based on an alignment with CYP102: structural studies on specific CYP2D6 substrate metabolism. Xenobiotica 1997;27:31939.

37 Beaune P, Pessayre D, Dansette P, et al. Autoantibodies against cytochromes P450: role in human diseases. Adv Phainst cytochromes P450: $1994 ; 30: 199-245$.

38 Pessayre D. Role of reactive metabolises in drug-induced hepatitis. F Hepatol 1995;23(suppl 1):16-24

39 Van Pelt FN, Straub P, Manns MP. Molecular basis of druginduced immunological liver injury. Semin Liver Dis 1995; 15:283-300

40 Lytton SD, Helander A, Zhang-Gouillon ZQ, et al. Autoantibodies against cytochromes P-4502E1 and P-4503A in alcoholics. Mol Pharmacol 1999;55:223-33.

41 Riley RJ, Smith G, Wolf CR, et al. Human anti-endoplasmic reticulum autoantibodies produced in aromatic anticonvulsant hypersensitivity reactions recognise rodent CYP3A proteins and a similarly regulated human P450 enzyme(s) proteins and a similarly regulated human P450 enzyme(s) published erratum appears in Biochem Biophys Res 1993;191:32-40.

42 Leeder JS, Gaedigk A, Lu X, et al. Epitope mapping studies with human anti-cytochrome P450 3A antibodies. Mol Pharmacol 1996;49:234-43. 
43 Winqvist $\mathrm{O}$, Gustafsson J, Rorsman F, et al. Two different cytochrome P450 enzymes are the adrenal antigens in autoimmune polyendocrine syndrome type I and Addison's disease. F Clin Invest 1993;92:2377-85.

44 Winqvist O, Karlsson FA, Kampe O. 21-Hydroxylase, a major autoantigen in idiopathic Addison's disease. Lancet 1992;339:1559-62.

45 Krohn K, Uibo R, Aavik E, et al. Identification by molecular cloning of an autoantigen associated with Addison's diseas as steroid 17 alpha-hydroxylase. Lancet 1992;339:770-3.

46 Muratori L, Zauli D, Giostra F, et al. LKM1 appearance in a HLA-DR3+ patient with chronic hepatitis C during interferon treatment. F Hepatol 1993;18:259-60.
47 Mackie FD, Peakman M, Yun M, et al. Primary and secondary liver/kidney microsomal autoantibody response following infection with hepatitis C virus. Gastroenterology 1994;106:1672-5.

48 Lohr H, Manns M, Kyriatsoulis A, et al. Clonal analysis of liver-infiltrating $\mathrm{T}$ cells in patients with LKM-1 antibodypositive autoimmune chronic active hepatitis. Clin Exp Immunol 1991;84:297-302.

49 Lohr HF, Schlaak JF, Lohse AW, et al. Autoreactive CD4+ LKM-specific and anticlonotypic T-cell responses in LKM-1 antibody-positive autoimmune hepatitis. Hepatology 1996;24:1416-21. 